\title{
Detection of Obstacles using Stereo Imaging
}

\author{
Sai P. Deshmukh \\ PG Student \\ Department of E\&TC \\ Engineering, \\ TSSM's Bhivarabai \\ Sawant College of Engineering \& Research, \\ Narhe, \\ SPPU, Pune, \\ Maharashtra, India
}

\author{
A.M. Deshpande \\ Assoc. Professor \\ Department of E\&TC \\ Engineering, \\ TSSM's Bhivarabai \\ Sawant College of Engineering \& Research, \\ Narhe, \\ SPPU, Pune, \\ Maharashtra, India
}

\begin{abstract}
This paper presents an implementation of algorithm for detecting obstacles based on stereo vision technique. This algorithm mainly focuses on detection of obstacle and computation of obstacle depth based on stereo matching and disparity map. This technique incorporates several steps such as pre-processing and depth analysis for obstacle detection. In the preprocessing step, Otsu's thresholding and adaptive thresholding methods are used for efficient detection of obstacles. After computation of obstacles, depth analysis is performed with the help of disparity map. Experimental results are demonstrated giving robust analysis of obstacle detection based on standard stereo dataset.
\end{abstract}

\section{Keywords}

stereo vision; obstacle detection; disparity map generation; depth computation

\section{INTRODUCTION}

Stereo vision is the field of Computer vision that has been heavily researched in recent years. Stereo vision is a technique from which one can extract 3D information and distance of a scene from at least two images (left and right), taken from different viewpoints [1], [2]. Pairs of images of a scene are taken by a digital camera that is moved between images. Stereo vision has several applications such as obstacle detection, image-based rendering, view synthesis etc. The given system mainly focuses on detecting obstacles using stereo vision technique.

Obstacle detection is one of the important steps for the applications such as autonomous navigation system. It is one of the most important parameters for an autonomous navigation system. Conventionally, obstacle detection system has to identify and compare the position of obstacles. For the obstacle detection using stereo imaging few parameters are very crucial viz., size, position of obstacle, accuracy and speed of the algorithm. This information may be obtained using the approaches such as ladar (laser ranging), sonar (sound ranging) or vision based techniques [3]. Stereo imaging technique provides advantages in terms of accurate position estimation as well as fast computation with low cost solution.

In this paper an algorithm for obstacle detection technique for tracking the obstacles in the auto-stereoscopic images is implemented. This technique uses adaptive thresholding and Otsu's methods for the purpose of detection of obstacle in the provided stereo images. Then, disparity is computed followed by computation of depth improving the efficiency of the obstacle detection. This algorithm has been examined by two methods for detecting obstacles, along with the development of graphical user interface (GUI) for this system.

The rest of the paper is organized as follows: In Section II, the related work on obstacle detection is summarized. The given algorithm implementation is discussed in Section III. The experimental results are demonstrated in Section IV. Finally, Section V concludes the paper.

\section{RELATED WORK}

An algorithm for detecting obstacle using stereo vision or stereo imaging detects an appropriate obstacle and estimates the depth of the obstacle using stereo matching technique and disparity map. There are many researchers who have proposed several techniques related to detection of obstacles.

An object collision detection algorithm based on disparity images is proposed in [3]. This method uses a 2D Ensemble Empirical Mode Decomposition image optimization algorithm and a two layer disparity image segmentation to detect nearby objects.

$\mathrm{Mu}-\mathrm{Chun} \mathrm{Su}$ et al. [4] derived an algorithm for stereo matching. By using fast cross correlation and rectangular subregioning (RSR), this technique produces stereo matching dense disparity map. The disparity map for the stereo images is found in the 3D correlation coefficient volume by obtaining the global 3D maximum-surface.

G. Balakrishnan et al. [5] proposed an algorithm where disparity is calculated with less computational efforts as compared to area based matching technique. This method is helpful for autonomous navigation for blind detecting obstacles in indoor environments [5]. It uses feature based technique for computation of disparity which leads to sparse disparity map compared to that of area based technique.

J. C. A. Fernandes et al. [6] proposed the algorithm to build a correction table for the video camera set-up in use, which remains almost constant in various industrial applications. This table divides the image in regions. For each region the correction can be put as a single delta- $\mathrm{x} /$ delta-y translation of the included pixels. This work is useful for setting up of two cameras with distortion-free arrangement in stereo vision based applications.

R. Danescu et al. [7] proposed a system for dynamic obstacle detection in driving environments. This system also provides real-time tracking solution for driving environments. Stereovision basis is used in this system to decide the obstacle grid and stereo reconstruction. 
In this paper simpler and effective thresholding (automatic and adaptive) approach is combined with the concept of stereo vision to compute the depth map and decide the accurate position of obstacles from two images.

\section{METHODOLOGY FOR OBSTACLE DETECTION}

The methodology used for obstacle detection in this paper is faster and reliable for detecting obstacles in indoor environments.

Evaluation of image geometry and camera parameters is the first step to rectify images captured from two cameras with equal focal lengths. This process is similar to that of camera calibration. A camera calibration process can be defined as a process of finding the true parameters of the camera that took photographs. Some of these parameters are focal length, principal point and lens distortion.

Next steps are pre-processing and obstacle detection. For obstacle detection, the adaptive thresholding and Otsu's thresholding methods are used. After these steps the disparity map and depth are computed. A flow diagram of complete obstacle detection method is shown in Fig.1.

Few basic assumptions as given in [8] are very useful for the automatic obstacle detection algorithm. These assumptions are as follows:

- Obstacles are assumed to be the objects which have some predefined depth from the surface

- Obstacles lie sufficiently higher from the ground surface

- Obstacles should be easily and quickly distinguishable from the background intensity in the image

\subsection{Evaluation of Image Geometry and Camera Parameters}

This process is carried out during camera calibration. Calibration is the first step to rectify images captured from two cameras with different focal lengths. This helps to have parallel and exact horizontal epipolar line. Details about camera parameters are given in [1]. For the stereoscopic arrangement of the cameras, camera calibration is an important step while capturing the input images in real-time.

In this paper, stereo images are obtained by setting up two cameras indoor as well as from the standard stereo dataset [16]. However, experimental results are provided only on standard stereo dataset.

\subsection{Obstacle Detection}

A good obstacle detection system, as suggested in [9], must be capable of the following:

- To detect obstacles on a given space in good time

- To detect and identify correct obstacles

- To identify and ignore ground features that may appear as obstacles

Experimental results show that the techniques used in given obstacle detection system i.e., an adaptive thresholding and Otsu's method satisfies the conditions mentioned above to a greater extent for indoor environment.

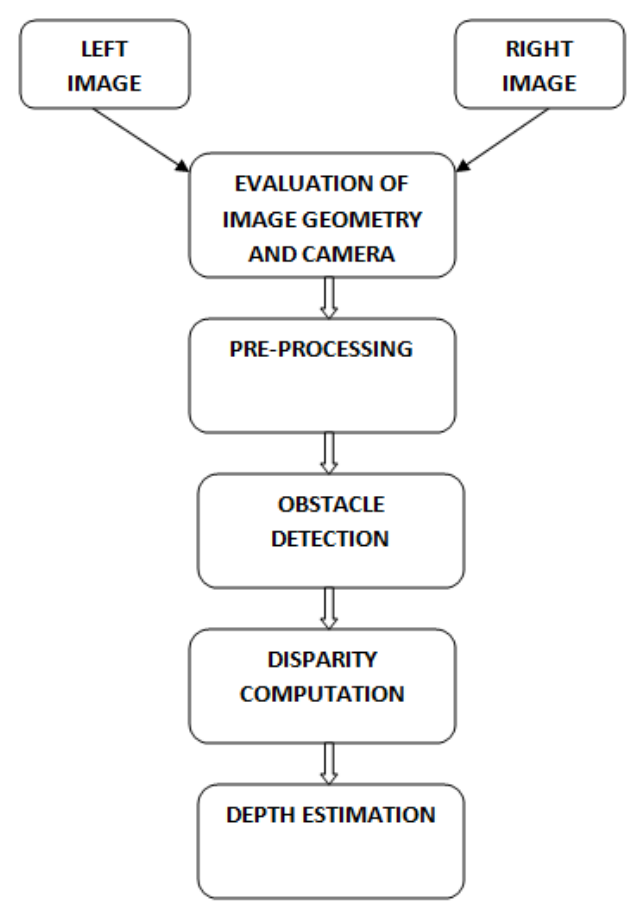

Fig.1 Flow diagram of obstacle detection method

\subsubsection{Adaptive Thresholding}

In the image thresholding, the image is segmented by setting some fixed threshold. Pixels having intensity values above the threshold will have foreground value and all the remaining pixels will have background value.

An adaptive thresholding is an approach whose input is either a grayscale image or a color image and gives a binary image as an output which represents the segmentation [10]. The implementation of the adaptive thresholding technique separates the foreground portions from the background with non-uniform illumination in the given image in which noise removal was also done by using mean and median filter. The adaptive thresholding is based on the average intensity of whole image [11].

The binary image is obtained as the output with the local threshold mean and/or median for the given input image. Another requirement for the implementation of this adaptive thresholding is the selection of local window size which is tested from $3 \times 3$ to $15 \times 15$ (Varying window size option is provided in the GUI of this system). After this pre-processing the disparity map and depth are computed [2].

\subsubsection{Otsu's Method}

Otsu's thresholding is a popular and easy to implement method. It gives effective thresholding results for bimodal intensity distribution of input [13]. It is a type of global thresholding. Otsu's method is aimed in finding the optimal value for the global threshold [12], [13]. An algorithmic approach for Otsu's method is given below:

1. Compute histogram and probabilities of each intensity level

2. Set up initial $\omega_{\mathrm{i}}(0)$ and $\mu_{\mathrm{i}}(0)$, where the $\omega$ and $\mu$ are the probabilities and class means respectively. 
3. Step through all possible thresholds $t=1 \ldots$ maximum intensity

a. Update $\omega_{\mathrm{i}}$ and $\mu_{\mathrm{i}}$

b. Compute $\sigma_{\mathrm{b}}^{2}(\mathrm{t})$, where $\sigma_{\mathrm{b}}^{2}$ is variance.

4. Desired threshold corresponds to the maximum $\sigma_{\mathrm{b}}^{2}(\mathrm{t})$

5. Compute two maxima (and two corresponding thresholds). $\sigma_{b 1}^{2}(t)$ is the greater maximum and $\sigma_{\mathrm{b} 2}{ }^{2}(\mathrm{t})$ is the greater or equal maximum

6. ${\text { Desired threshold }=\frac{\text { threshold }_{1}+\text { threshold }_{2}}{2}}_{2}$

\subsection{Disparity Computation}

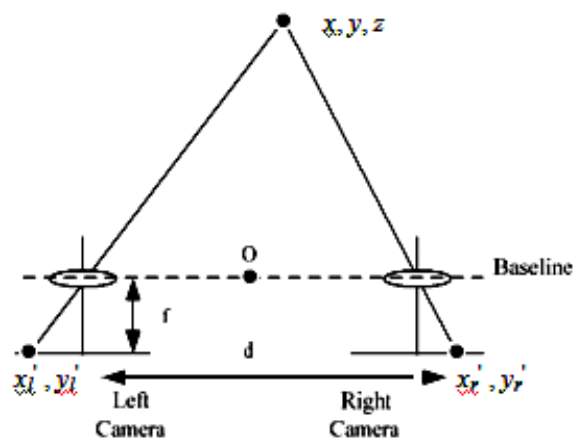

Fig. 2 Disparity and depth computation- a geometrical view [14]

For computation of disparity map of given stereoscopic images (the left and right image) the stereo matching algorithm is used so as to obtain a rectified stereo image pair [1].

Fig. 2 illustrates the overall idea of stereo vision camera arrangement for disparity and depth computation. Here, $\mathrm{xl}$ and $\mathrm{yl}$ are the coordinates of left image, $\mathrm{xr}$ and $\mathrm{yr}$ are the coordinates of the right image, $f$ is the focal length, $d$ is the distance between two images [14].

Disparity computation can be done by using feature based algorithm and area based algorithm. The given system uses area based algorithm for the computation of a disparity map. Feature based algorithm may have sparse disparity map, whereas, an area-based algorithm has the advantage of having dense disparity map, which is ideal for indoor and outdoor environments and easy to implement [2]. Hence, it uses areabased algorithm.

Stereo matching is done by using SHD (Sum of Hamming Distance) SAD (Sum of Absolute Distances), Normalized Cross Correlation (NCC), Zero Normalized Cross Correlation (ZNCC) functions matching with $9 * 9$ windows. This algorithm is also known as block matching algorithm [1], [2], [15].

SHD, SAD, NCC and ZNCC terms between two images can be computed as given below [15]:

$$
\begin{aligned}
& \text { SHD }=\sum \mathrm{I}_{1}(\mathrm{i}, \mathrm{j}) \text { bitXOR } \mathrm{I}_{2}(\mathrm{i}+\mathrm{x}, \mathrm{j}+\mathrm{y}) \\
& \mathrm{SAD}=\sum_{\mathrm{i}, \mathrm{j} \in \mathrm{w}}\left|\mathrm{I}_{1}(\mathrm{i}, \mathrm{j})-\mathrm{I}_{2}(\mathrm{i}+\mathrm{x}, \mathrm{y}+\mathrm{j})\right| \\
& \mathrm{NCC}=\frac{\sum_{\mathrm{i}, j \in \mathrm{w}} \mathrm{I}_{1}(\mathrm{i}, \mathrm{j}) \mathrm{I}_{2}(\mathrm{i}+\mathrm{x}, \mathrm{j}+\mathrm{y})}{\sqrt{\sum_{\mathrm{i}, \mathrm{j} \in \mathrm{w}} \mathrm{I}_{1}^{2}(\mathrm{i}, \mathrm{j}) \mathrm{I}_{2}^{2}(\mathrm{i}+\mathrm{x}, \mathrm{j}+\mathrm{y})}}
\end{aligned}
$$

$$
\operatorname{ZNCC}=\frac{\sum_{\mathrm{i}, \mathrm{j} \in \mathrm{w}}\left(\mathrm{I}_{1}(\mathrm{i}, \mathrm{j})-\overline{\mathrm{I}}_{1}(\mathrm{i}, \mathrm{j})\right) \cdot\left(\mathrm{I}_{2}(\mathrm{i}+\mathrm{x}, \mathrm{j}+\mathrm{y})-\overline{\mathrm{I}}_{2}(\mathrm{i}+\mathrm{x}, \mathrm{j}+\mathrm{y})\right)}{\sqrt{\sum_{\mathrm{i}, \mathrm{j} \in \mathrm{w}}\left(\mathrm{I}_{1}(\mathrm{i}, \mathrm{j})-\overline{\mathrm{I}}_{1}(\mathrm{i}, \mathrm{j})\right)^{2} \cdot\left(\mathrm{I}_{2}(\mathrm{i}+\mathrm{x}, \mathrm{j}+\mathrm{y})-\overline{\mathrm{I}}_{2}(\mathrm{i}+\mathrm{x}, \mathrm{j}+\mathrm{y})\right)^{2}}}
$$

where, I1 and $\mathrm{I} 2$ are left and right images, $(\mathrm{i}, \mathrm{j})$ are the pixels of window $w,(i+x),(j+y)$ are the pixels of right image I2.

Depth map can be achieved by using disparity computation. This can be done by considering square window of certain size (as it's a block matching technique) around the pixel of interest in the reference image. By comparison one can easily search the homologous pixel within the window in the target image, while moving along the corresponding scan line.

The main aim of the disparity computation is to search the correlated pixel within a certain disparity range " $\mathrm{d}$ " (where $\mathrm{d}$ $\mathrm{E}[0, \ldots, \mathrm{dmax}])$. The value of $d$ should be in such a range that, the produced disparity map contains maximum similarity and minimum errors associated with it [15]

\subsection{Depth Computation}

Finally depth of obstacle is computed as follows:

$$
z(x, y)=\frac{f . . B}{d(x, y)}
$$

where $\boldsymbol{f}$ is the focal length, $\boldsymbol{B}$ is the base line (distance between two cameras centre), $\boldsymbol{d}(\boldsymbol{x}, \boldsymbol{y})$ is disparity value and $z(x, y)$ is depth value in $\mathrm{x}$ row and y column [1], [2]

\section{EXPERIMENTAL RESULTS}

The given obstacle detection algorithms are implemented in MATLAB. The experimental results for obstacle detection by using Otsu's method and adaptive thresholding method are compared by testing 50 images from standard Middlebury stereo dataset [16], on the basis of estimated time to detect obstacles.

Fig. 3 shows the GUI developed for the obstacle detection algorithm. Out of 50 test images, results obtained for 4 images viz., 'baby.png', 'stand.png', 'ball.png' and 'flowerpot.png' are shown in Fig. 4.

A comparative evaluation of obstacle detection methods on the basis of estimated time for detecting obstacle is shown in Table 1 . Table 2 gives corresponding results of disparity and depth computation.

Table 1. Estimated time for detecting obstacles

\begin{tabular}{|c|c|c|c|}
\hline \multirow{2}{*}{ Test image } & \multirow{2}{*}{$\begin{array}{c}\text { Obstacle } \\
\text { Detected }\end{array}$} & \multicolumn{2}{|c|}{$\begin{array}{c}\text { Estimated Time in } \\
\text { Seconds }\end{array}$} \\
\cline { 3 - 4 } & & $\begin{array}{c}\text { Adaptive } \\
\text { Thresholding }\end{array}$ & $\begin{array}{c}\text { Otsu's } \\
\text { Method }\end{array}$ \\
\hline baby.png & $\begin{array}{c}\text { human } \\
\text { being }\end{array}$ & 0.06196 & 7.1705 \\
\hline stand.png & net & 0.1504 & 7.8714 \\
\hline ball.png & ball & 0.52221 & 9.3655 \\
\hline flowerpot.png & pot & 0.05481 & 7.2382 \\
& & & \\
\hline
\end{tabular}


Table 2. Computation of disparity and depth values

\begin{tabular}{|c|c|c|c|}
\hline Test image & $\begin{array}{c}\text { Disparity } \\
\text { Computation } \\
\text { Method }\end{array}$ & $\begin{array}{c}\text { Disparity } \\
\text { Value }\end{array}$ & $\begin{array}{c}\text { Depth } \\
\text { Value }\end{array}$ \\
\hline \multirow{3}{*}{ baby.png } & SHD & 4.7104 & 529.91 \\
\cline { 2 - 4 } & SAD & 4.6754 & 533.88 \\
\cline { 2 - 4 } & NCC & 4.8569 & 513.93 \\
\cline { 2 - 4 } & ZNCC & 4.5427 & 549.48 \\
\hline \multirow{3}{*}{ stand.png } & SHD & 4.8318 & 516.60 \\
\cline { 2 - 4 } & SAD & 4.7765 & 522.58 \\
\cline { 2 - 4 } & NCC & 4.7744 & 522.81 \\
\cline { 2 - 4 } & ZNCC & 4.5327 & 550.691 \\
\hline
\end{tabular}

\begin{tabular}{|c|c|c|c|}
\hline \multirow{3}{*}{ ball.png } & SHD & 5.2887 & 471.974 \\
\cline { 2 - 4 } & SAD & 5.2310 & 477.179 \\
\cline { 2 - 4 } & NCC & 4.9279 & 506.531 \\
\cline { 2 - 4 } & ZNCC & 4.6316 & 538.931 \\
\hline \multirow{3}{*}{ flowerpot.png } & SHD & 5.4489 & 458.099 \\
\cline { 2 - 4 } & SAD & 5.4158 & 460.897 \\
\cline { 2 - 4 } & NCC & 4.9206 & 507.286 \\
\cline { 2 - 4 } & ZNCC & 4.5349 & 550.601 \\
\hline
\end{tabular}

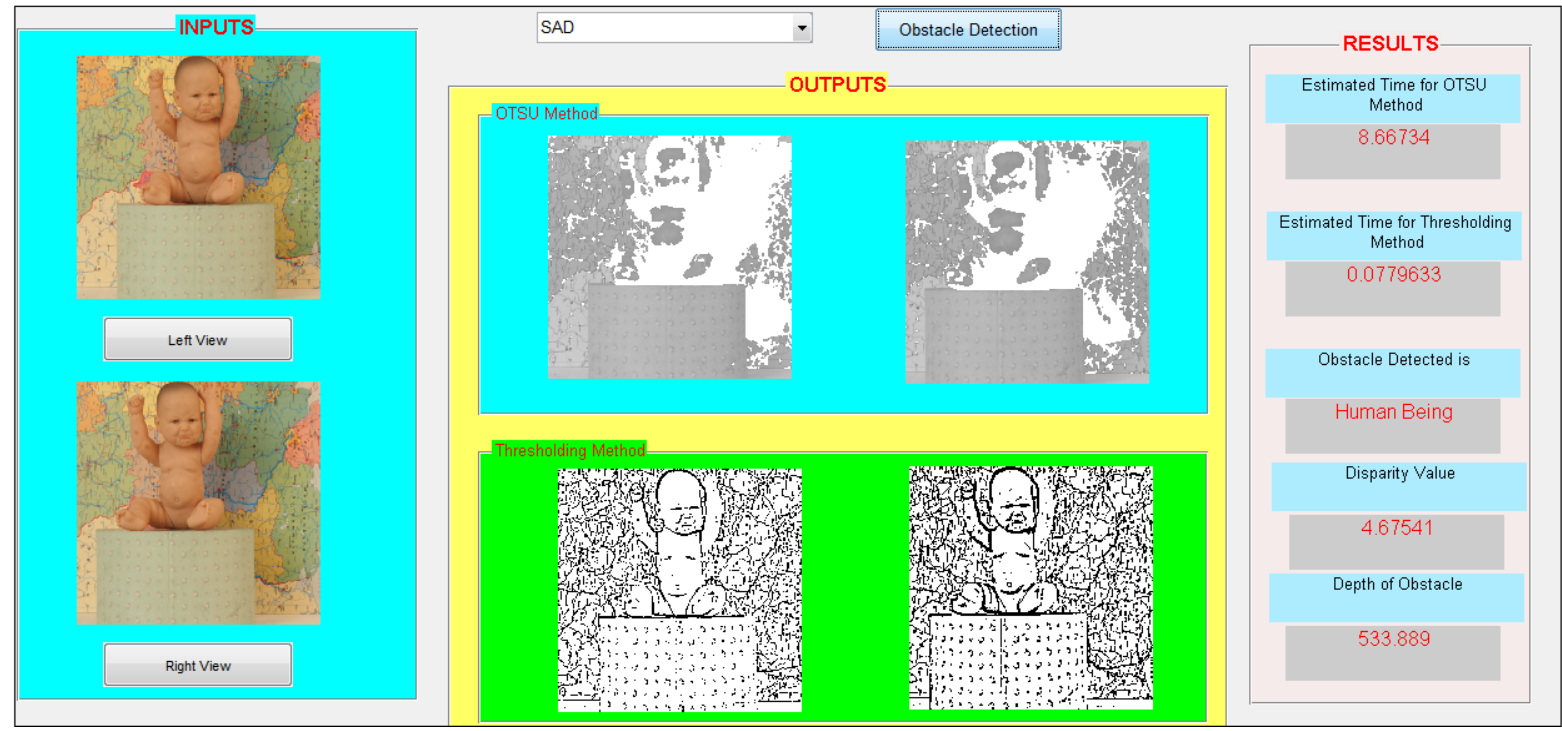

Fig. 3 GUI developed for obstacle detection method
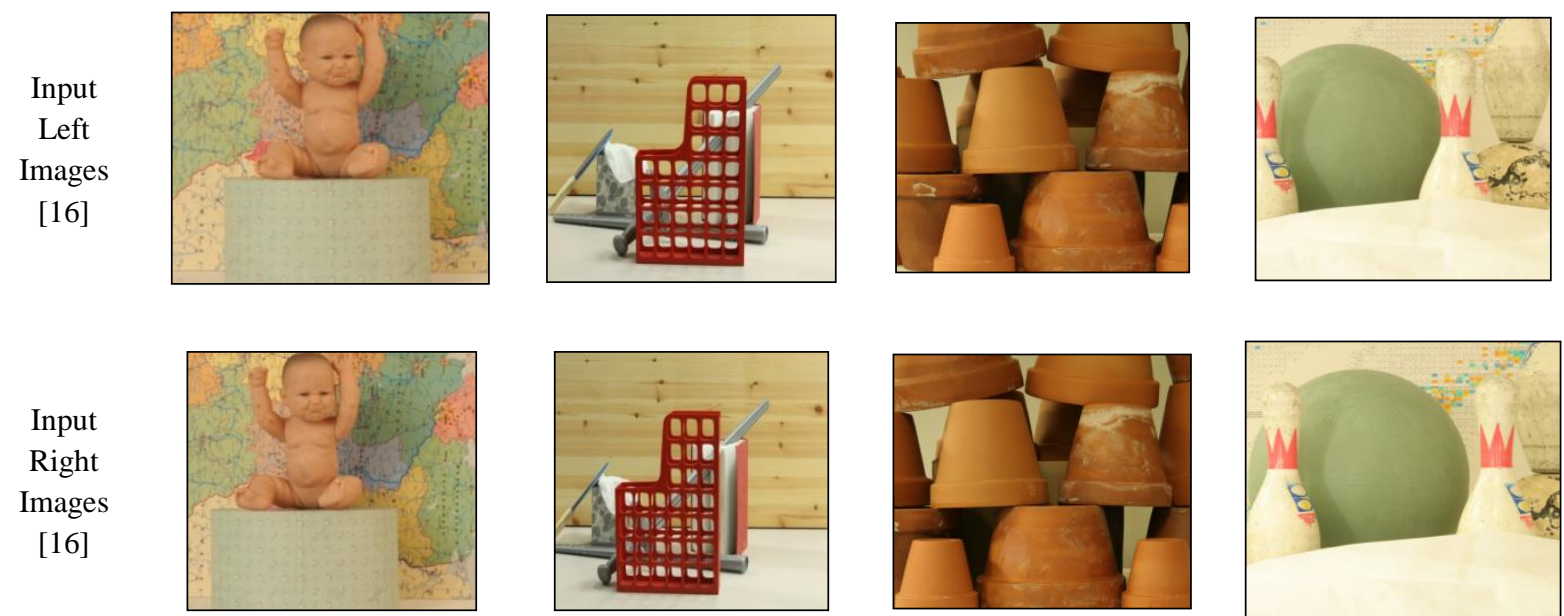

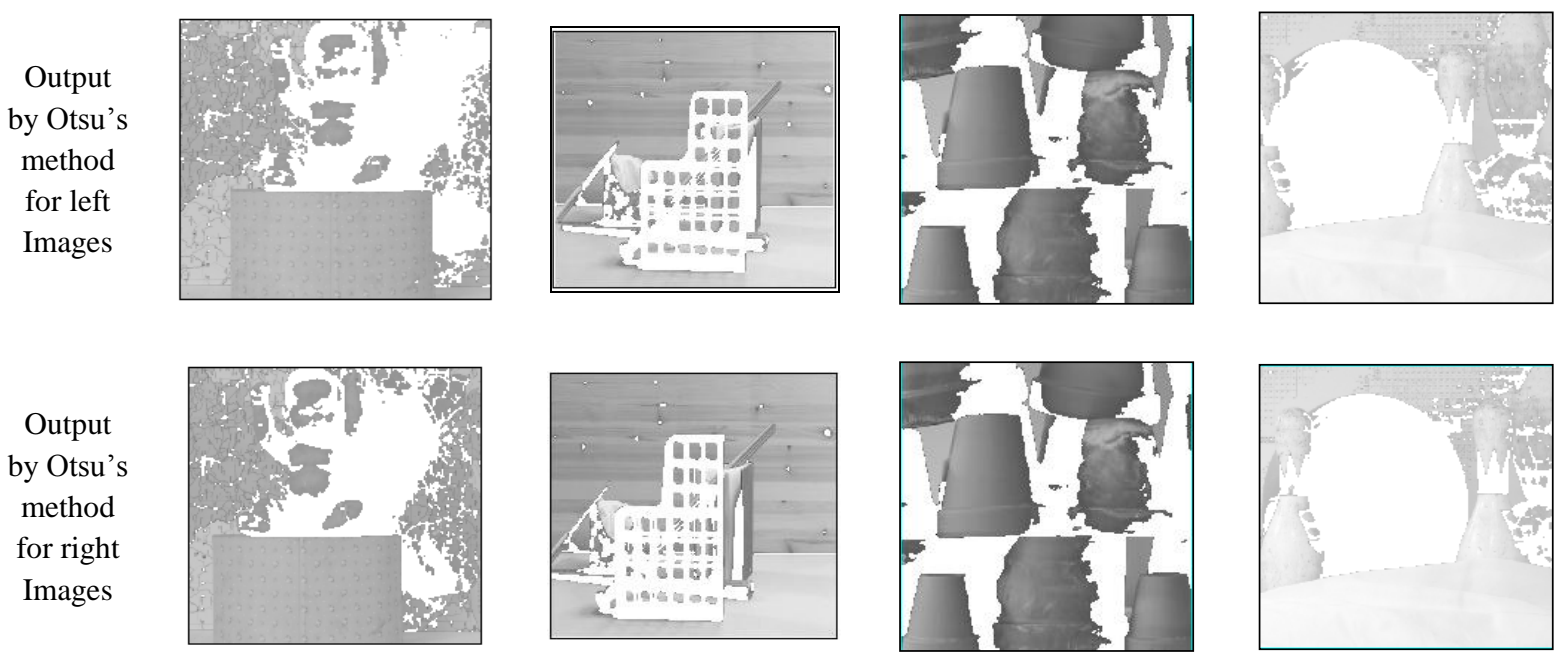

Output
by
Adaptive
Threshol
ding for
left
image
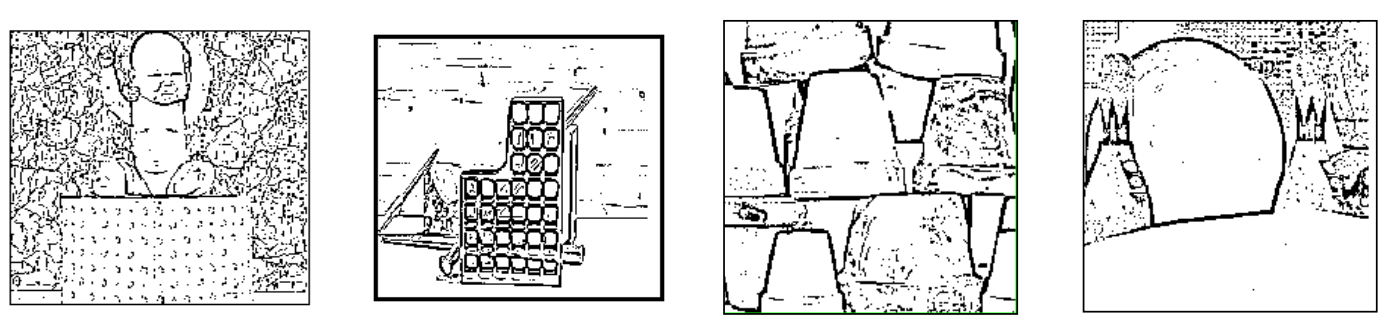

Output
by
Adaptive
Threshol
ding for
right
image
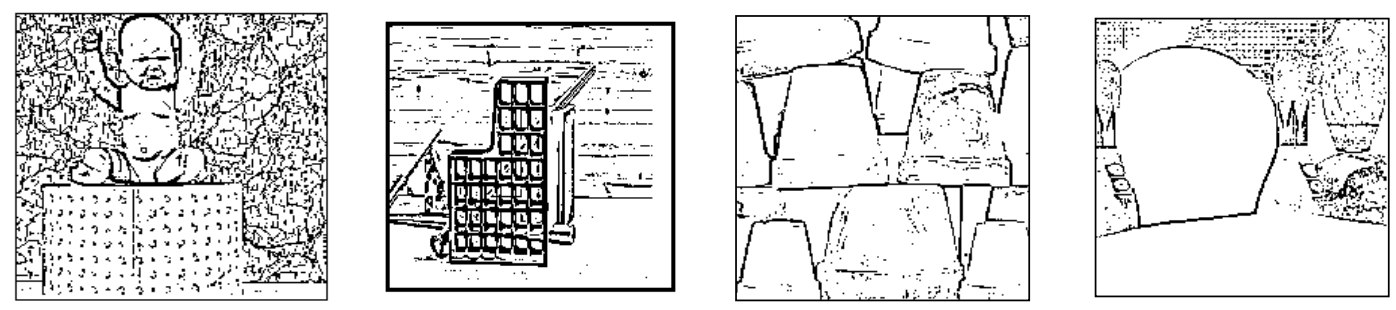

Fig. 4 Results of obstacle detection method. First Row: Input left images; Second row: Input right images; Third Row: Result of Otsu's method of left images; Fourth Row: result of Otsu's method of right images; Fifth Row: Result of adaptive thresholding of left images; Sixth Row: Result of adaptive thresholding of right images

\section{CONCLUSIONS AND FUTURE SCOPE}

Stereo vision based obstacle detection and computing obstacle depth is implemented in this paper. It is performed using stereo matching technique, disparity map computation and depth estimation. Two methods are used for detecting obstacles i. e., adaptive thresholding and Otsu's Method. The comparative analysis of these two methods on the basis of estimated time for obstacle detection proves that adaptive thresholding is more efficient than Otsu's method. It requires less computational time.

An area-based algorithm for stereo matching is performed on the basis of SHD (Sum of Hamming Distance), SAD (Sum of Absolute Distance), NCC (Normalized Cross Correlation), ZNCC (Zero Normalized Cross Correlation) functions matching with $9 * 9$ window. Thus, depth analysis is done with respect to all these stereo matching techniques. A comparative evaluation for estimated depths is done by using all these methods. It shows that the disparity computation achieves desired level of computation of depth. Hence with the depth information one can easily detect position of obstacle in the given stereo pair image. Thus given system can efficiently detect the obstacle along with its depth computation which will be useful for autonomous navigation assistance system.
6. REFERENCES

[1] Forsyth A. and Ponce J., Computer Vision: A modern approach, New Jersey, Prentice-Hall, 2002.

[2] S. Fazli, H. M. Dehnavi, P. Moallem, "A Robust detection Method in Highly Textured Environments using Stereo Vision", Second International Conference on Machine Vision, IEEE Computer Society, pp. 97-100, Dec. 2009.

[3] Paulo Costa, Hugo Fernandes, Paulo Martins, João Barroso, "Obstacle detection using stereo imaging to assist the navigation of visually impaired people", Procedia Computer Science, 14(2012) 83-93, ELSEVIER Science, 2012.

[4] Mu-Chun Su, Yi-Zeng Hsieh, and Yu-Xiang Zhao, "A Simple Approach to Stereo Matching and Its Application in Developing a Travel Aid for the Blind", in Proceedings of the 2006 Joint Conference on Information Sciences, Department of Computer Science \& Information Engineering, National Central University, Taiwan, R.O.C.

[5] G. Balakrishnan, G. Sainarayanan, R. Nagarajan, Sazali Yaacob, "Stereopsis Method for Visually Impaired to 
Identify Obstacles Based on Distance", Third IEEE International Conference on Image and Graphics, pp. 580-583, Dec. 2004

[6] J.C.A. Fernandes, M.J.O. Ferreira and J.A.B.C. Neves, "Fast Correction of Lens Distortion for Image Applications", in Proceedings of the IEEE International Symposium on Industrial Electronics, ISIE'97, pp. 708 712, vol.2, July 1997.

[7] Radu Danescu, Cosmin Pantilie, Florin Oniga, and Sergiu Nedevschi, "Particle Grid Tracking System Stereo-vision Based Obstacle Perception in Driving Environments", IEEE Intelligent Transportation Systems Magazine, Vol. 4, Issue 1, pp. 6-20, 2012.

[8] Kowalik, R., Kwasniewski, S., "Navigator - A Talking GPS Receiver for the Blind", Lecture Notes in Computer Science 3118, pp. 446-449, Springer Berlin / Heidelberg (2004)

[9] Syedur Rahman, "Obstacle Detection for Mobile Robots Using Computer Vision”, Final Project, March 2005.

[10] http://homepages.inf.ed.ac.uk/rbf/HIPR2/adpthrsh.htm
[11] Shivani Thakur, Virender Sharma and Amit Chhabra, "A Review: Obstacle Tracking Using Image Segmentation', International Journal of Innovative Research in Computer and Communication Engineering, vol. 2, no. 6 , pp. 4590-4596, June 2014.

[12] Miss. Hetal J. Vala, Prof. Astha Baxi, A Review on Otsu Image Segmentation Algorithm, International Journal of Advanced Research in Computer Engineering \& Technology (IJARCET) Volume 2, Issue 2, February 2013.

[13] Nobuyuki Otsu, "A Threshold Selection Method from Gray-Level Histograms", IEEE Transactions On Systems, Man, And Cybernetics, Vol. Smc-9, No. 1, January 1979.

[14] P. Foggia, Jean-Michel Jolion, A. Limongiello, M. Vento, "Stereo Vision for Obstacle Detection: A Graph-Based Approach", Graph-Based Representations in Pattern Recognition 2007, LNCS 4583, pp. 37-48.

[15] https://siddhantahuja.wordpress.com/tag/sum-ofabsolute-differences-sad/

[16] http://cat.middlebury.edu/stereo/data.html 